\title{
Some Theorems for Real Increasing Functions in Elementary Fixed Point Theory
}

\author{
Maria Luigia Diviccaro, Salvatore Sessa* \\ Dipartimento di Architettura, Università di Napoli, Napoli, Italy \\ Email: *sessa@unina.it
}

How to cite this paper: Diviccaro, M.L. and Sessa, S. (2020) Some Theorems for Real Increasing Functions in Elementary Fixed Point Theory. Advances in Pure Mathematics, 10, 501-507.

https://doi.org/10.4236/apm.2020.109031

Received: May 27, 2020

Accepted: September 7, 2020

Published: September 10, 2020

Copyright $\odot 2020$ by author(s) and Scientific Research Publishing Inc. This work is licensed under the Creative Commons Attribution International License (CC BY 4.0).

http://creativecommons.org/licenses/by/4.0/

\section{Abstract \\ We obtain some theorems for real increasing functions showing that elemen- tary fixed point theory can bring to astonishing results by assuming only a few properties, some of which intrinsically possessed from these functions. An application is given for a theorem of quasi-compactness and a known re- sult in posets is also recalled and applied to real intervals. \\ Keywords \\ Increasing Function, Upper Broad Sequentially Semicontinuity on the Right, Greatest Fixed Point}

\section{Introduction}

Notwithstanding some words of the title, Elementary Fixed Point Theory [1] does not mean to establish "elementarily" fixed point theorems in the context of "structurally simple spaces" such as metric spaces or Banach spaces, but to deduce fixed point theorems from some intrinsic properties of the selfmaps involved and thus we arrive at astonishing results, like in the case of this paper where we consider the set $\boldsymbol{F}\left(x_{0}\right)$ of selfmaps functions $f$ of $\boldsymbol{R}$ (the reals) having a given point $x_{0} \in \boldsymbol{R}$ as the fixed point of $f$, i.e. $f\left(x_{0}\right)=x_{0}$. Furthermore, we consider the subset $G\left(x_{0}\right)$ of $F\left(x_{0}\right)$ formed from functions $f$ such that

$$
x_{0} \leq f(x)<x \text { for any } x>x_{0}
$$

and

$$
x<f(x) \leq x_{0} \text { for any } x<x_{0}
$$

Moreover, let $H\left(x_{0}\right)$ be the subset of $F\left(x_{0}\right)$ of functions $f$ "attracting" $R$, which means the sequence $f^{n}(x) \rightarrow x_{0}$ as $n \rightarrow \infty$ for any $x \in \boldsymbol{R}$, being $f^{\prime}(x)$ the $n$-th iterate of $f$ defined usually by $f^{1}(x)=f(x), f^{n+1}=f\left(f^{n}(x)\right)$ for any integer positive $n$. In general, this term is transferred to a contractive function in 
the sense of, historically speaking, a theorem of Banach-Caccioppoli [2] [3] and other famous authors (see, e.g., those cited in the good books [1] [4]), have a unique fixed point $x_{0}$ "attracting the whole metric space". Inspired from an old paper of Sgambati [5], we establish some results for a real increasing function (often considered as selfmap of a compact interval of $\boldsymbol{R}$ ).

We start with the following theorem.

Theorem 1. Let $f \in \boldsymbol{G}\left(x_{0}\right)$ be increasing. If

for any $x>x_{0}$, there exists a $y>x$ such that $f(t) \leq x$ for $\left.t \in\right] x, y[$

and

for any $x<x_{0}$, there exists a $y<x$ such that $f(t) \geq x$ for $\left.t \in\right] y, x[$,

then $f \in \boldsymbol{H}\left(x_{0}\right)$.

Proof. We limit to prove the theorem under (1.3) (the proof under (1.4) is similar).

The thesis is obvious if $f^{n}(x)=x_{0}$ for some $n$ since if $f$ is increasing and $f \in \boldsymbol{G}\left(x_{0}\right)$, we have $x>f(x)>\cdots>f^{n}(x)=x_{0}=f^{n+p}(x)$ for $p=1,2, \cdots$. Then we can assume $f^{n}(x)>x_{0}$ for any integer positive $n$. By (1.1) and since $f$ is increasing, we have that

$$
x_{0}=f\left(x_{0}\right)<f^{n+1}(x)<f^{n}(x) \text { for any integer positive } n \text { and for any } x>x_{0}
$$

that is the sequence $\left\{f^{p}(x)\right\}$ is strictly decreasing and hence converges to the least upper bound $r$ as $n \rightarrow \infty$. Then for any $\varepsilon>0$, there exists some integer positive $s$ such that $f^{n}(x)<r+\varepsilon$ for any $n \geq s$. We deduce $f^{n}(x)>r$ for $n=1,2, \cdots$ from (1.5). Assume that $r>x_{0}$. By (1.3), there exists $y>r$ such that $f(t) \leq r$ for $t \in] r, y[$ and choose an $\varepsilon=y-r$. So there exists some integer positive $m$ such that $f^{n}(x)<y$ for any $n \geq m$ and then we should have, in particular, $r<f^{m}(x) \leq r$ which is a contradiction. Therefore $r=x_{0}$.

Claim. The condition (1.3) or (1.4) is not necessary in order to have the belongness of a function $f \in \boldsymbol{F}\left(x_{0}\right)$ to the set $\boldsymbol{H}\left(x_{0}\right)$. Indeed, let any $x_{0} \in \boldsymbol{R}$ and consider a strictly decreasing sequence $\left\{h_{n}\right\}$ of rationals convergent to some rational $x_{1}>x_{0}$. Hence it is enough to define the function $f\left(h_{n}\right)=\pi \cdot\left(h_{n}-x_{1}\right)+x_{1}$ (irrational value) and $f(x)=x_{0}$ if $x \neq h_{n}$ for any positive integer $n$ in order to obtain a function $f$ which proves such assertion.

Remark 1. Let $f \in \boldsymbol{F}\left(x_{0}\right)$ and $f$ be increasing. Then it is immediate to verify (1.3) and (1.4) are equivalent, respectively, to the following: $x>x_{0}$ implies either $\lim _{y \rightarrow x^{+}} f(y)<x$ or there exists a $y>x$ such that $f(t)=x$ for $t \in] x, y[$

and

$x<x_{0}$ implies either $\lim _{y \rightarrow x^{+}} f(y)>x$ or there exists a $y<x$ such that $f(t)=x$ for $t \in] y, x[$ 
Since an increasing function which has the property $\lim _{y \rightarrow x^{+}} f(y)<x$ (resp., $\lim _{y \rightarrow x^{+}} f(y)>x$ ) implies having the property $f(x)<x$ (resp., $f(x)>x$ ), then we can enunciate the following theorem and its corollary due to the author of [5] without proof:

Theorem 2. An increasing function $f \in \boldsymbol{F}\left(x_{0}\right)$ belongs to $H\left(x_{0}\right)$ iff $\left(x>x_{0}\right.$ implies $f(x)<x$ and the second member of (1.6) holds) and ( $x<x_{0}$ implies $f(x)>$ $x$ and the second member of (1.7) holds).

Corollary 3. A strictly increasing function $f \in \boldsymbol{F}\left(x_{0}\right)$ belongs to $H\left(x_{0}\right)$ iff $\lim _{y \rightarrow x^{+}} f(y)<x$ for any $x>x_{0}$ and $\lim _{y \rightarrow x^{+}} f(y)>x$ for any $x<x_{0}$.

The following theorem and its corollary, due to the author of [5], are also easily proved:

Theorem 4. In order to have the belongness of a function $f \in \boldsymbol{F}\left(x_{0}\right)$ to the set $H\left(x_{0}\right)$, it is enough that $f$ is right upper semicontinuous in every $x>x_{0}$ and left lower semicontinuous in every $x<x_{0}$.

Proof. Indeed a function $f \in \boldsymbol{F}\left(x_{0}\right)$ which is right upper (resp., left lower) semicontinuous verifies (1.3) (resp., (1.4)).

Corollary 5. In order to have the belongness of a continuous function $f \in \boldsymbol{F}\left(x_{0}\right)$ to the set $H\left(x_{0}\right)$, it is enough to satisfy (1.1) and (1.2).

\section{Other Results}

The following lemma is useful for the successive Theorems 7 and 8 .

Lemma 6. Let $a>0$ and $f$ be an increasing selfmap of the compact interval [0, a] having zero as unique fixed point. Then $f(x)<x$ for any $x \in(0, a]$.

Proof. We have that $f(a)<a$. If $f(x)>x$ for some $x \in(0, a)$, we should have that there striction $g$ of $f$ to the compact interval $[x, a]$ is an increasing selfmap of $[x, a]$ itself because $x<g(x)=f(x) \leq g(t)=f(t) \leq g(a)=f(a)<a$ for any $t \in(x, a)$ holds. Then, e.g., by applying a famous theorem of Tarski [6] to an increasing selfmap $g$ of the compact interval $[x, a]$, minimum and maximum fixed points of $g$ should exist in $[x, a]$, which should be also fixed points of $f$ in $[0, a]$, a contradiction to the hypothesis.

Theorem 7. Let $a>0$ and $f$ be an increasing selfmap of the compact interval $[0, a]$ having zero as unique fixed point and $x \in(0, a)$ implies either $\lim _{y \rightarrow x^{+}} f(y)<x$ or there exists an $y>x$ such that $f(t)=x$ for $\left.t \in\right] x, y[$. Then we have that $f^{n}(x) \rightarrow 0$ as $n \rightarrow \infty$ for any $x \in[0, a]$.

Proof. Lemma 6 assures that $f(x)<x$ for any $x \in(0, a]$, so we can extend the function $f$ from $[0, a]$ to an increasing selfmap $g$ of $\boldsymbol{R}$ in the following way: $g(x)=0$ if $x \leq 0, g(x)=f(x)$ if $x \in(0, a), g(x)=f(a)$ if $a \leq x$ which satisfies (1.1) and (1.2) for $x_{0}=0$. By invoking Theorem 2, we have $g^{n}(x)=f^{n}(x) \rightarrow 0$ for any $x \in[0, a]$.

Theorem 8. Let $a>0$ and $f$ be an increasing selfmap of the compact interval $[0, a]$ having zero as unique fixed point and right continuous in any $x \in(0, a)$. Then we have that $f^{n}(x) \rightarrow 0$ as $n \rightarrow \infty$ for any $x \in[0, a]$. 
Proof. By Lemma 6, we have that $f(x)<x$ for any $x \in(0, a]$. Since $f$ is right continuous, we also have that $\lim _{y \rightarrow x^{+}} f(y)<x$ for any $x \in(0, a)$. So the thesis comes from Theorem 7.

As application of above Theorem 7, we give a generalization of a theorem of "quasi compactness" due to J. Einsenfeld and V. Lakshmikantham [7]. We recall that a selfmap $\psi$ of a bounded metric space $A_{0}$ is defined quasi compact if the sequence of measures of non-compactness $\left\{\gamma\left(A_{n}\right)\right\}$ of the closed subsets of $A_{0}$ recursively given by $A_{n+1}=c l\left(\psi\left(A_{n}\right)\right)$ for $n=0,1,2, \cdots$ converges to zero as $n \rightarrow \infty$.

The following theorem holds:

Theorem 9. Let be a selfmap $\psi$ of a bounded metric space $A_{0}$. Suppose that $a>\gamma\left(A_{0}\right)$ and there exists an $f$ be an increasing selfmap of the compact interval $[0, a]$ having zero as unique fixed point. Further $x \in(0, a)$ implies either $\lim _{y \rightarrow x^{+}} f(y)<x$ or there exists a $y>x$ such that $f(t)=x$ for $\left.t \in\right] x, y[$. Then $\psi$ is quasi compact provided that for any subset $A$ of $A_{0}$, we have that

$$
\gamma(\psi(A)) \leq f(\gamma(A))
$$

Proof. From (2.1), it follows that $\gamma\left(A_{n}\right) \leq f^{n}\left(\gamma\left(A_{0}\right)\right)$ for any $A_{n+1}=c l\left(\psi\left(A_{n}\right)\right), n=0,1,2, \cdots$. By Theorem 7 , we have that $f^{n}\left(\gamma\left(A_{0}\right)\right) \rightarrow 0$ as $n \rightarrow \infty$ and hence the thesis.

Remark 2. J. Einsenfeld and V. Lakshmikantham [7] assume all the hypothesis of Theorem 9 except that $\mathrm{f}$ is considered right continuous in any $x \in(0, a)$. Thus our Theorem 9 is an extension of that result since a right continuous function satisfies the hypothesis contained in Theorem 9.

\section{Upper Broad Sequentially Semicontinuity on the Right}

The following theorem is widely known (e.g., [6]. We give a short proof.

Theorem 10. Let $\mathrm{f}$ be an increasing and right continuous selfmap of a compact interval $X$ of $\boldsymbol{R}$ and there exists a point $x_{0} \in X$ such that $f\left(x_{0}\right) \leq x_{0}$. Then the limit $z$ of the sequence $\left\{f^{\prime}\left(x_{0}\right)\right\}$ is the greatest fixed point of $f$ in $S_{-}\left(x_{0}\right)=\left\{x \in X: x \leq x_{0}\right\}$.

Proof. $z$ is a fixed point of $f$ in $S_{-}\left(x_{0}\right)$ since f is right continuous. If $w=f(w)$ is another fixed point of $f$ in $S_{-}\left(x_{0}\right)$, being $f$ increasing, we have that $f^{n}(w)=w \leq f^{n}\left(x_{0}\right)$ for any integer positive $n$ and then $w \leq z$ as $n \rightarrow \infty$.

We provide a generalization of Theorem 10 starting with the following definition:

Definition 11. Let $f$ be a selfmap of subset $X$ of $R$. We say that $f$ is upper broad sequentially semicontinuous on the right at the point $x \in X$ if for any decreasing sequence $\left\{x_{n}\right\}$ converging to $x$ and such that is convergent the sequence $\left\{f\left(x_{n}\right)\right\}$ to the limit $l$, there exists a non-negative integer $k=k(x)$ for which we have that $f^{k}(l) \leq f^{k+1}(x)$. We say that $f$ is upper broad sequentially semicontinuous on the right on $X$ if $f$ is upper broad sequentially semicontinuous on the right at any point $x \in X$. 
Remark 3. Any right continuous selfmap $f$ of an interval $X$ of $R$ is upper broad sequentially semicontinuous on the right on $X$ because $k(x)=0$ for any $x \in X \quad$ (we naturally understand that $f^{0}(x)=x$ ).

Definition 12. Let $f$ be a selfmap of interval $X$ of $R$ and let $\mathrm{f}$ be an upper broad sequentially semicontinuous on the right at the point $x \in X$. We define upper broad sequentially semicontinuity on the right parameter of a decreasing sequence $\left\{x_{n}\right\}$ converging to $x$ and such that is convergent the sequence $\left\{f\left(x_{n}\right)\right\}$ to the limit $l$, denoted by $k=k\left(x,\left\{x_{n}\right\},\left\{f\left(x_{n}\right)\right\}\right)$ for abuse of notation, the minimum of the set $\left\{p\right.$ non-negative integer: $\left.f^{p}(l) \leq f^{p+1}(x)\right\}$.

Theorem 13. Let $f$ be a selfmap of a compact interval $X$ of $R$ and $f$ be an increasing and upper broad sequentially semicontinuous on the right over $X$. Moreover there exists in $X$ a point $x_{0}$ such that $f\left(x_{0}\right) \leq x_{0}$ and let

$k=k\left(x_{0},\left\{f^{n}\left(x_{0}\right)\right\},\left\{f^{n+1}\left(x_{0}\right)\right\}\right)$ be the upper broad sequentially semicontinuity on the right parameter of the decreasing sequence $\left\{f^{f}\left(x_{0}\right)\right\}$. Then, if $z$ is the limit of the sequence $\left\{f^{f}\left(x_{0}\right)\right\}, f^{k}(z)$ is the greatest fixed point of $f$ in $S_{-}\left(x_{0}\right)=\left\{x \in X: x \leq x_{0}\right\}$.

Proof. For brevity, let $x_{n}=f^{n}\left(x_{0}\right)$ for any non-negative integer, we have $f^{k}(z) \leq f^{k+1}(z)$ by definition 12. On the other hand, $z \leq x_{n}$ because $\left\{x_{n}\right\}$ converges to its least upper bound, from which $f(z) \leq f\left(x_{n}\right)=x_{n+1}$ because $f$ is increasing and hence $f(z) \leq z$, from it follows that $f^{k+1}(z) \leq f^{k}(z)$. Therefore $f\left(f^{k}(z)\right)=f^{k}(z)$. If $w=f(w)$ is another fixed point of $f$ in $S_{-}\left(x_{0}\right)$, we have that $f^{n}(w)=w \leq f^{n}\left(x_{0}\right)=x_{n}$ and then $w \leq z$ as $n \rightarrow \infty$. Since $\mathrm{f}$ is increasing, we have $f^{k}(w)=w \leq f^{k}(z)$.

By Remark 3, Theorem 13 is an effective extension of Theorem 10. In fact Theorem 13 holds in the following example where Theorem 10 is not applicable:

Example 14. Let $f$ be a selfmap of $[0,1]$ be defined as $f(x)=x$ if $x \in[0, a], 1 / 2>a>0, \quad f(x)=a \quad$ if $\quad x \in[a, 1 / 2], \quad f(x)=x / 2+1 / 4 \quad$ if $x \in(1 / 2,1]$. It is easily seen that $f$ is right continuous in $[0,1]-\{1 / 2\}$, and hence upper broad sequentially semicontinuous on the right because of Remark 3 and also at $1 / 2$ because $k\left(1 / 2,\left\{f^{n}(1 / 2)\right\},\left\{f^{n+1}(1 / 2)\right\}\right)=1$. Indeed $f$ is not right continuous at $1 / 2$ and hence Theorem 10 is not applicable but it can easily see that $f^{p}(1 / 2)=a$ is the greatest fixed point in $S_{-}(1 / 2)$ for any positive integer $p$ with $1 \leq p$.

\section{A Simple Result in Posets}

The next result holds in a partially ordered set (poset) but it can hold in subsets of $R$ as we show in a suitable example. This result can be deduced also from our previous works [8] [9] but we prefer to give a proof for making self-contained the present paper.

Theorem 15. Let $(X, \leq)$ be a poset and $\mathrm{f}$ be an increasing selfmap of $X$. Assume the existence of a point $x_{0}$ such that $x_{0} \leq f\left(x_{0}\right)$ and the following condition holds:

(C1) if $C$ is a chain which has not a supremum in $X$, there exists a positive in- 
teger $1 \leq n$ such that there exists the supremum of the chain $f^{f}(C)$, denoted by $\sup f^{\prime}(C)$.

Then $\mathrm{f}$ has a fixed point $z$ in $S^{+}\left(x_{0}\right)=\left\{x \in X: x_{0} \leq x\right\}$.

Proof. Consider the subset of $\mathrm{X}$ defined by $Y=\left\{x \in X: x_{0} \leq x \leq f(x)\right\} . Y$ is no empty because there at least $x_{0} \in Y$ by hypothesis. Let $C$ be a chain of $Y$ and assume that there no exists its supremum in $X$, then there exists $y=\sup f^{n}(C)$ for some positive integer $1 \leq n$.

So, from $x_{0} \leq c \leq f(c)$ for any $c \in \boldsymbol{C}$, we deduce that $x_{0} \leq c \leq f(c) \leq f^{2}(c) \leq \cdots \leq f^{n}(c) \leq y$ for any $c \in C$ since $f$ is increasing, that is $y$ is an upper bound of $C$ in $X$. But we claim that $y$ lives in $Y$. Indeed, $x_{0} \leq c \leq f^{n-1}(c) \leq y$ for any $c \in \boldsymbol{C}$, then $x_{0} \leq c \leq f^{n}(c) \leq f(y)$ for any $c \in \boldsymbol{C}$ due to the increasingness of $f$, i.e. $f(y)$ is an upper bound of the chain $f^{\prime}(C)$, which means $x_{0} \leq y \leq f(y)$, i.e. $y \in Y$ and thus any chain $C$ of $Y$ has an upper bound in $Y$. By Zorn lemma, $Y$ has a maximal element $z$ such that $z \leq f(z)$ and therefore $z=f(z)$, i.e. the thesis.

We illustrate Theorem 15 with a suitable example:

Example 16. Let $X=[0,2]-\{1 / 2\}$ with usual ordering and $\mathrm{f}$ be defined as $f(x)=(x+1) / 2$ if $x \in[0,1]-\{1 / 2\}$ and $f(x)=x$ if $x \in[1,2]$. Then $f$ is increasing and the unique chains of $X$ not having supremum in $X$ are the chains with supremum $1 / 2$ which does not belong to $X$.

Note that $x \leq f(x)$ for any $x \in X$ and $\sup f^{n}(\boldsymbol{C}) \in X$ for any integer $n$ such that $1 \leq n$. Indeed, if $C$ is a chain included in $[0,1 / 2)$, then $f(C)$ is included in $(1 / 2,1]$ and $f(\boldsymbol{C})=\boldsymbol{C}$ if included in $[1,2]$. Note that $z=1$ if $x_{0} \in[0,1]-\{1 / 2\}$ and $z=x_{0}$ if $x_{0} \in[1,2]$.

\section{Conclusion}

We have proved various theorems for real increasing functions $f$ giving the new concept of upper broad sequentially semicontinuity on the right at a point $x$ and related parameter $k$ of a decreasing sequence $\left\{x_{n}\right\}$ convergent to $x$ and such that also converges the sequence $\left\{f\left(X_{n}\right)\right\}$. Naturally, a dual concept like lower broad sequentially semicontinuity on the left and related parameter $k$ of an increasing sequence $\left\{X_{n}\right\}$ at a point $x$, such that also converges the sequence $\left\{f\left(X_{n}\right)\right\}$, holds as well, however it should be interesting to establish geometrical and topological properties of the parameter $k$, here not investigated for brevity.

\section{Acknowledgements}

In memoriam of the masterly friend Prof. Antonio Zitarosa (Napoli).

\section{Authors' Contributions}

The authors read and approved the final manuscript.

\section{Conflicts of Interest}

The authors declare to have no competing interests. 


\section{References}

[1] Subrahmanyam, P.V. (2018) Elementary Fixed Point Theorems. Springer Nature Singapore Pte Ltd. https://doi.org/10.1007/978-981-13-3158-9

[2] Banach, S. (1922) Sur les Operations dans les Ensembles Abstraits et Leur Application aux Equations Integrales. Fundamenta Mathematicae, 3, 133-181. https://doi.org/10.4064/fm-3-1-133-181

[3] Cacciopoli, R. (1932) Sugli elementi uniti delle trasformazioni funzionali: Un agreee di esistenza e di unicità ed alcune sue applicazioni. Rendiconti del Seminario Matematicodella Università di Padova, 3, 1-15.

http://www.numdam.org/item/RSMUP $1932 \quad 3 \quad 1 \quad 0 /$

[4] Kirk, W.A. and Sims, B. (2001) Handbook of Metric Fixed Point Theory. Kluwer Academic, London. https://doi.org/10.1007/978-94-017-1748-9

[5] Sgambati, L. (1971) Sulle Applicazioni di R in R Dotate di un Punto Unito Attraente. R. Rend. Ist. Matem. Univ. di Trieste, 3, 62-74. https://rendiconti.dmi.units.it/node/4/400pm

[6] Tarski, A. (1955) A Lattice-Theoretical Fix Point Theorem and Its Applications. Pacific Journal of Mathematics, 5, 285-309. https://doi.org/10.2140/pjm.1955.5.285

[7] Eisenfeld, J. and Lakshmikantham, V. (1977) Remarks on Non-Linear Contraction and Comparison Principle in Abstract Cones. Journal of Mathematical Analysis and Applications, 61, 116-121. https://doi.org/10.1016/0022-247X(77)90147-0

[8] Diviccaro, M.L. and Sessa, S. (2008) Common Fixed Points of Increasing Operators in Posets and Related Semilattice Properties. International Mathematical Forum, 3, 2123-2128.

[9] Diviccaro, M.L. and Sessa, S. (2013) Maximum and Minimum Fixed Points of Isotone Operators in Partially Ordered Sets. International Journal of Contemporary Mathematical Sciences, 8, 69-74. https://doi.org/10.12988/ijcms.2013.13007 